\title{
Design of the high precision liquid level measuring system
}

\author{
Hong Ma \\ (Nanjing Institute of Industry Technology,Nanjing 210023,China) \\ mh_purple@163.com
}

Keywords:sensor,liquid level control,data acquisition

Abstract.According to the application demand of the present water supply system of the Internet of things, designing the high precision liquid level measure and transmit circuit,especially,for the requirements of pressure measuring range and linear precision, using for pressure measurement and control of modern buildings and intelligent water supply system.

\section{Introduction}

It's necessary to measure and control the liquid level of reservoir,wide application in manufacture and life. The measurement of liquid level can actually be converted to the measurement of depth pressure. The water supply capacity of modern intelligent building and intelligent water supply system is very important. In the design of the system, it's necessary to measure and transfer data of the pressure and liquid level, etc.For example, in the metallurgical industry, the control device of metallurgical electrolyte flow and liquid level, requires very high precision of measurement and control. The liquid level control system shall be specially designed, including the liquid level circuit of reservoir, liquid level control circuit, liquid level detection circuit of reservoir, control liquid level detection circuit, information processing control circuit,open and close control circuit, water pump, such as signal mapping (signal conversion), which can improve the sensitivity of controlled and control, reducing the measurement time delay.

One of the key issues we need to solve the intelligent requirements of water supply system and liquid level measurement, with the concept of Internet of things, are getting higher and higher. There are many ways to test various parameters of reservoir, however, a lot of measuring instrument can be quite hard to accept because of the price,installation,environment, the existing liquid level measuring instrument(such as the controlled float by liquid level measuring instrument,often fails because of its size and environment which limits its application). The measurement precision,data transmission and processing of the existing liquid level measuring instrument can't adapt to rapid development of the construction industry requirements of present,on the whole, especially the demand of intelligent.

This paper presents the design of high precision measurement and transmission circuit of liquid level pressure, according to the application requirements of the water supply system and Internet of things of intelligent buildings, etc,which especially fit in with the requirements of large measuring range and high linear accuracy, can be used to pressure measurement and level control of modern buildings and intelligent water supply system, can also be used to monitor and control the pressure of fire tank and monitor the pressure at the bottom of reservoir dam. 


\section{The overall hardware design of the system}

The system uses $51(\operatorname{mc} 9 \mathrm{~s} 12 \times 32)$ single chip microcomputer as the control core, designing a system to monitor the liquid level. The characteristic of the control system is visualized display and controlled the liquid level freely. Host hardware circuit consists of 5 modules, namely, data acquisition module, host circuit and communication interface module, analog input channel module, analog output channel module, software module. The power supply system of this design can be realized by the new power supply technology, such as the hybrid Photovoltaic-Wind Generation Technology, etc, and wireless mode by making improvements to the two development port.The overall hardware design diagram of the system is shown in figure 1.

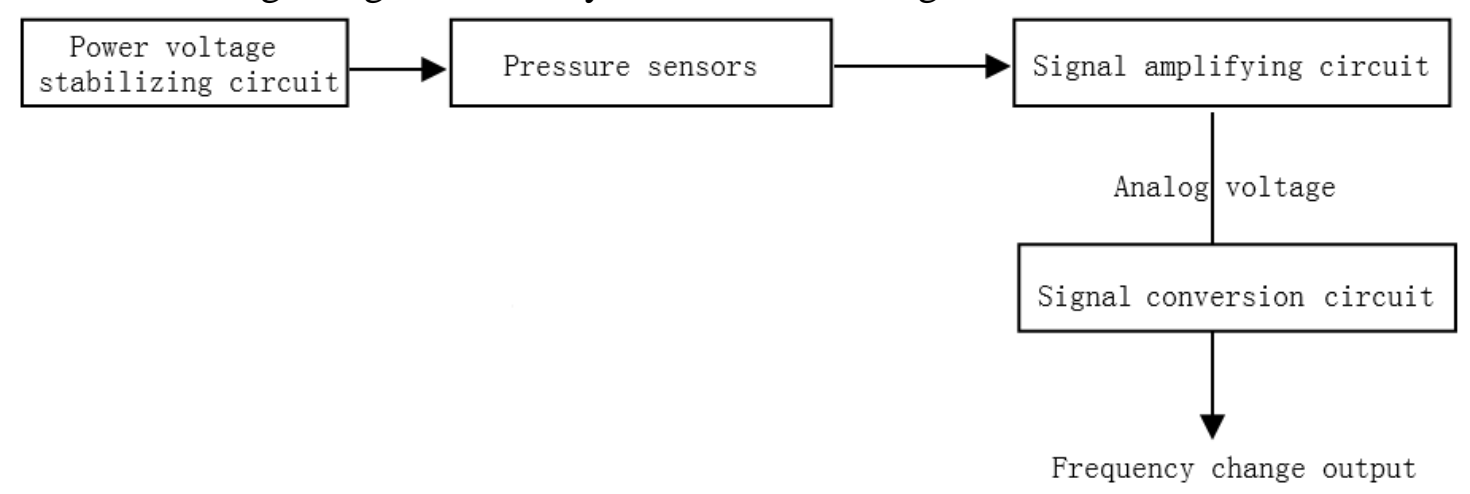

Fig.1 The overall hardware design diagram of the system

The overall characteristics of the system include the following aspects:

(1) Selecting the pressure sensor with good sensitivity,large measuring range and good stability,the range of pressure is up to 1-15Psig.

(2) Using the linear voltage regulator and operational amplifier, keeping the working voltage of the circuit at high stability and high precision, so as to achieve the purpose of high-precision measurement.

(3) Applying the bridge amplifier is composed of matched operational amplifier to amplify the output of the pressure sensor, which is suitable for remote control and transmission.

(4) In order to adapt to long distance transmission, the analog voltage of $0-5 \mathrm{~V}$ is converted to $0-5$ $\mathrm{kHz}$ frequency output. The circuit adopts temperature compensation to further improve the stability.

\section{Liquid level control design}

Through data processing and data acquisition,according to the characteristics of the monitoring objects, converting the simulation of voltage and current into digital signal,through the 485 output, real-time detecting the liquid level of reservoir, and comparing with the preset value, reaching the detection value of liquid level.If the detection value is higher than the upper limit, water pump stops supplying water; if the detection value is lower than the lower limit, water pump starts to supply water. The measurement value is displayed in real time to realize monitoring the water level of the liquid level of reservoir.

Working process of liquid level control is when the liquid level of reservoir changes, pressure sensor converts the variation into voltage signal;which amplified by the operational amplifier circuit into a range of $0 \sim 5 \mathrm{~V}$ standard signal, and then send into $\mathrm{A} / \mathrm{D}$ converter which converts the analog signals into digital signals, and real-time data acquisition, and processing, and control output according to requirements set, and display the liquid level. The characteristic of the control system 
is visual display, and can control the liquid level arbitrarily. The working principle block diagram is shown in figure 2.

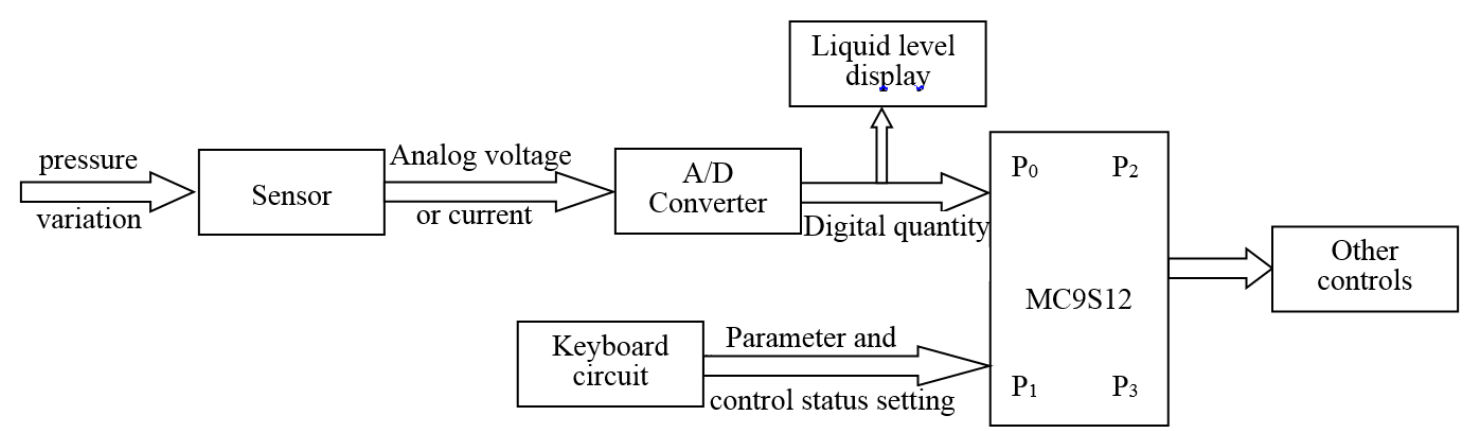

Fig. 2 Principle block diagram of liquid level control

\section{Data acquisition modules}

The core technology of this design is data processing and data acquisition module, data acquisition module of the focus in the design and selection of the sensor,the design uses a bridge type load pressure sensor, which adopts stainless steel welding beam mechanical structure, good sealing performance, anti-corrosion, anti pollution, widely used in various industries, suitable for harsh occasions in particular. The principle is shown in Figure 3. The 4 resistors stick to the elastomer to form a full bridge balance detection circuit. When the sensor subjected to external load, elastic strain, the bridge arm is out of balance, under the influence of external bridge supply ,the bridge outputs unbalanced DC voltage signals, which directly proportional to the load magnitude of the sensor, thus enabling the conversion of the force to electricity.

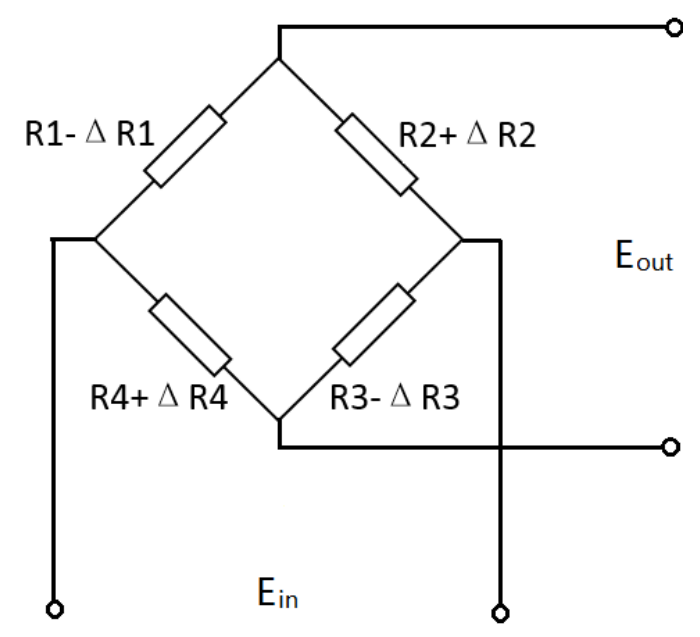

Fig. 3 Bridge load pressure sensor

\section{Conclusion}

The high precision liquid level measuring system, from needs of the social and market, according to the application characteristics of the Internet of things, using amplifier of high stability and temperature compensation measures, designing circuit of the sensor system with wide range, high precision, can adapt to measure pressure of reservoir, and designed the converter circuit about voltage and frequency,the output voltage of liquid level pressure measurement converted into the frequency change, high sensitivity, reducing the measurement time delay,also improving the 
resistance against external interference and disturbances ,meeting the need for long-distance transmission. The system has many advantages, such as the range of hydraulic pressure measurement is large,high accuracy, good stability, the output signal transmission distance over other similar systems, has very high potential market value in the application of the Internet of things.

\section{References}

[1]Texas Instruments Incorporated $\quad$ MSP430F42XO MIXED SIGNAL MICROCONTROLLER[EB].2005 .

[2]Mouritsen J . A Survey on NC System Simulation Tracking and Feedback Control Techniques[J] , IRE Transactions , $2006,23(1):$ 78-94 .

[3]Ron Helson . The Hart Book[M] . Hampshire : GGH Marketing Communication Ltd , 2001 .

[4]Sipex Corporation. SP6200 / 6201 Micropower, 100mA and 200mA CMOS LDO Regulators[EB] , 2006 .

[5]Romilly Bowden . HART Field Communications Protocol(Second Edition)[M] , FISHERROSEMOUNT , 1996 .

[6]Adrian Fernandez . Getting Started with the MSP430 LaunchPad[M] . Newnes , 2013.

Name: Hong Ma, F, 1979.12 birth. Nanjing people. Nanjing Institute of Industry Technology Institute of Energy and Electrical Engineering Lecturer, research direction: digital communications.

Address: JiangSu NanJing XianLin university city Cheng Pin Cheng 19 building room 506, unit 3; Code: 210023

Phone : 13951886232 (mobile) 